positive; $45.7 \%$ ( $\mathrm{N}=21$ ) were RF-ELISA positive and RF-nephelometry negative and $4.3 \%(N=2)$ the opposite, without a statistical significance.

Conclusions: ELISA is superior to nephelometry detecting RF in patients with $\mathrm{RA}$, as also in quantifying high-positive values.

References:

[1] Routine measurement of $\operatorname{lgM}, \lg G$, and $\lg A$ rheumatoid factors: high sensitivity, specificity, and predictive value for rheumatoid arthritis. Swedler W, Wallman J, Froelich CJ, Teodorescu M. J Rheumatol, 1997 Jun;24(6):1037-44.

[2] Is rheumatoid factor still a superior test for the diagnosis of rheumatoid arthritis? Singh U, Vishwanath A, Verma PK et al. Rheumatol int, 2010 June; 30(8):1115-9.

Disclosure of Interest: None declared

DOI: 10.1136/annrheumdis-2017-eular.2606

\section{AB1044 EVALUATION OF A FLUOROENZYME IMMUNOASSAY (ELIA-CTD) IN THE SCREENING OF PATIENTS SUSPECTED FOR AUTOIMMUNE CONNECTIVE TISSUE DISEASES}

M. Elkhalifa ${ }^{1}$, R. Abdulhadi ${ }^{1}$, H. Ramadan ${ }^{1}$, S. Barrientos ${ }^{1}$, S. Saleh ${ }^{1}$, O. Alsaed ${ }^{2}$, A.-W. Al-Allaf ${ }^{2} .{ }^{1}$ Laboratory Medicine and Pathology; ${ }^{2}$ Medicine, Hamad Medical Corporation, Doha, Qatar

Background: Detection of auto-antibodies directed against nuclear antigens (antinuclear antibodies or ANA) have important diagnostic and prognostic implications in connective tissue diseases (CTD). The conventional indirect immunofluorescence assay on HEp-2 cell line (ANA-IIF) is the most commonly used method to detect ANA. The ANA-IIF can be labor intensive and suffers from lack of specificity.

Objectives: To evaluate the utility of a new fluoroenzyme Immunoassay "EliACTD" as an alternative for screening patients suspected for autoimmune connective tissue diseases.

Methods: Sixteen Hundread (1600) consecutive patients' sera submitted for anti-nuclear antibodies were tested using the ANA-IIF (Diasorin S.P.A, Saluggia, Italy) and the new EliA-CTD screen (Phadia GmbH, Frieiburg, Germany). ANA testing was ordered by both primary and secondary care physicians. The EliACTD screening assay is a fluoroenzyme immunoassay which is performed on the Phadia-250 automated platform. The EliA-CTD assay contains ANA-targeted recombinant antigens including dsDNA, Sm-D, Rib-P, PCNA, U1-RNP (70, A, C), SS-A/Ro, SS-B/La, Centromere B, Scl-70, Fibrillarin, RNA Polymerase III, Jo-1, Mi-2, and PM-scl. The test results are expressed as ratio, with $>1.0$ considered positive. For ANA-IIF, the cut off for positive results was 1:40 or greater. Additionally, further testing for dsDNA and other extractable nuclear antigens (ENA) was undertaken on a subset of sera that were ANA-IIF+ or whenever there was discrepancy between the two methods.

Results: The overall agreement between the two methods was $84.2 \%$. Three hundread and eight (308) out of $1600(19.3 \%)$ samples tested positive by ANA-IIF positive as compared to $101 / 1600(6.6 \%)$ for the EliA-CTD assay. Additional testing showed that 105 samples were positive for ENA including dsDNA. Of those, 101 were EliA-CTD positive and 81 were ANA-IIF positive. By incorporating the ENA results, the calculated sensitivity and specificity for the EliA-CTD were $97.1 \%$ and $99.7 \%$ respectively with positive and negative predictive values for the EliA-CTD assay of $96.1 \%$ and $99.8 \%$, respectively. The corresponding sensitivity, specificity, positive predictive value (PPV) and negative predictive value (NPV) for the ANA-IIF assay at different dilutions is shown below:

\begin{tabular}{lcccc}
\hline Titer & Sensitivity (\%) & Specificity (\%) & PPV (\%) & NPV (\%) \\
\hline$\geq 1: 40$ & 77.7 & 84.8 & 26.0 & 98.2 \\
$\geq 1: 80$ & 60.3 & 95.3 & 32.4 & 98.4 \\
$\geq 1: 160$ & 57.4 & 97.4 & 41.3 & 98.4 \\
$\geq 1: 320$ & 46.5 & 98.7 & 48.8 & 98.5 \\
\hline
\end{tabular}

Conclusions: The new automated EliA-CTD assay shows superior sensitivity and specificity compared to the conventional labor intensive ANA-IIF. The EliACTD can be used as an upfront screening tool for connective tissue diseases. Depending on the clinical details, any EliA-CTD positive results could be reflexly followed by additional testing including ANA-IIF testing to elucidate the titer and pattern.

References:

[1] Hayashi N, Kawamoto T, Mukai M, Morinobu A, Koshiba M, Kondo S, Maekawa S, Kumagai S. Detection of Antinuclear Antibodies by Use of an Enzyme Immunoassay with Nuclear HEp-2 Cell Extract and Recombinant Antigens: Comparison with Immunofluorescence Assay in 307 Patients. Clinical Chemistry 2001; 47(9):1649-1959.

Disclosure of Interest: None declared

DOI: 10.1136/annrheumdis-2017-eular.2679

\section{AB1045 MACROPHAGE TARGETED POSITRON EMISSION TOMOGRAPHY (PET) FOR THE IMAGING OF INFLAMMATORY ARTHRITIS; AN IN VIVO AND IN VITRO INVESTIGATION OF TRANSLOCATOR PROTEIN (TSPO) TRACER UPTAKE}

N. Narayan ${ }^{1}$, H. Mandhair ${ }^{1}$, C. Coello ${ }^{2}$, A. Saleem ${ }^{2}$, A. Sabokbar ${ }^{1}$, P. Taylor ${ }^{1}$.
${ }^{1}$ Nuffield Dept of Orthopaedics, Rheumatology, Musculoskeletal Sciences, University of Oxford, Oxford; ${ }^{2}$ Imanova Centre for Imaging Sciences, London, United Kingdom

Background: TSPO targeted PET tracers are increasingly recognised as cellular imaging markers of macrophage infiltration, due to the high expression of TSPO on activated macrophages. Previous work demonstrated the ability of $\left[{ }^{11} \mathrm{C}\right] \mathrm{PK} 11195$ TSPO PET to detect subclinical inflammation in RA, and predict flare in both those with established RA and ACPA positive arthralgia. However, high background uptake of $\left[{ }^{11} \mathrm{C}\right] \mathrm{PK} 11195$ in bone, and inability of $\left[{ }^{11} \mathrm{C}\right] \mathrm{PK} 11195$ to detect lesions in AS has driven the investigation of newer TSPO tracers for the detection of inflammatory arthritis.

Objectives: Here, we present data confirming the ability of the TSPO tracer $\left.{ }^{11} \mathrm{C}\right] \mathrm{PBR} 28$ to detect and quantify synovitis in both RA and PsA, and in vitro work that assesses more fully what TSPO tracer accumulation in inflamed synovium actually reflects at a cellular level, especially considering TSPO is ubiquitously expressed.

Methods: 10 patients ( 5 with RA, 5 with PsA) with evidence of inflammation in one or both knees (as confirmed by clinical examination and US) and 4 healthy volunteers underwent PET/CT both knees using the TSPO tracer $\left[{ }^{11} \mathrm{C}\right] \mathrm{PBR} 28$. Arthritis patients underwent synovial biopsy of one knee within 7 days of scan. Healthy synovium was obtained from patients undergoing knee arthroscopy for ligamentous injury. Synovial tissue was stained for CD68, CD163 and TSPO. For in vitro work, human monocytes, lymphocytes and synovial FLS from RA patients were harvested, and macrophages differentiated from monocytes. RNA was extracted for PCR. Other cells underwent density centrifugation to extract the cytoplasmic cell fraction, and a radioligand binding assay with $\left[{ }^{3} \mathrm{H}\right] \mathrm{PBR} 28$ was undertaken, to assess tracer binding to TSPO in each cell type

Results: Tracer uptake correlated significantly with severity of inflammation on clinical examination and ultrasound, as did synovial sublining staining for CD68, TSPO and CD163 (see table 1). There was negligible staining for all stains in healthy control synovium. qPCR demonstrated highest TSPO mRNA in stimulated FLS (fold change 62.75 \pm 10.03 ) and M2 macrophages $(60.69 \pm 2.38$ ), with lymphocytes having the least TSPO expression. PBR28 saturation binding confirmed these findings at protein level (see graph 1).

\begin{tabular}{lccc}
\hline Tissue marker & \multicolumn{3}{c}{ Clinical synovitis severity } \\
\cline { 2 - 4 } & 1 (mild) & 2 (moderate) & 3 (severe) \\
\hline TSPO & $1.50 \pm 0.08$ & $2.19 \pm 0.19$ & $3.06 \pm 0.12$ \\
CD68 & $2.17 \pm 0.3$ & $2.42 \pm 0.21$ & $2.96 \pm 0.05$ \\
CD163 & $1.61 \pm 0.03$ & $2.19 \pm 0.1$ & $2.81 \pm 0.04$ \\
\hline
\end{tabular}

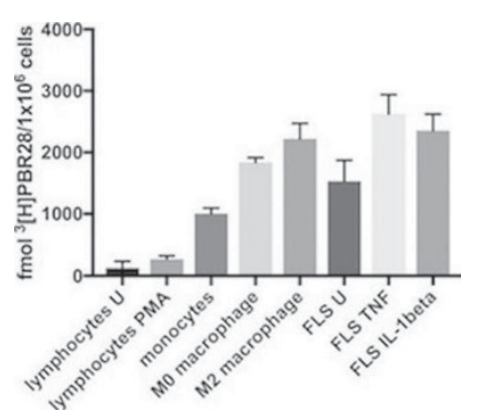

Conclusions: Our data demonstrates that the TSPO tracer PBR28 is capable of detecting and quantifying synovitis in RA and PsA. PBR28 tracer uptake correlates with macrophage marker staining, but not with fibroblast marker staining in our patient cohort. mRNA and protein data demonstrate, however, that there is a similar expression of TSPO in activated macrophages and activated FLS, hence TSPO tracer accumulation is as likely to represent FLS activation as it is macrophage activation.

References:

[1] Gent $Y Y$ et al. Macrophage positron emission tomography imaging as a biomarker for preclinical rheumatoid arthritis: findings of a prospective pilot study. Arthritis Rheum 2012; 64:62-6.

[2] Gent YYJ et al. Subclinical synovitis detected by macrophage PET, but not $\mathrm{MRI}$, is related to short-term flare of clinical disease activity in early RA patients: an exploratory study. Arthritis Res Ther 2015; 17:266.

Disclosure of Interest: None declared

DOI: 10.1136/annrheumdis-2017-eular.1838

\section{AB1046 COULD SERUM TWEAK LEVEL BE AN INDICATOR OF SUBCLINICAL ATHEROSCLEROSIS IN RHEUMATOID ARTHRITIS?}

M. Ucer ${ }^{1}$, N. Yilmaz ${ }^{2}$, B. Cengiz Elcioglu ${ }^{3}$, T. Sahin ${ }^{3}$, P. Gun Atak ${ }^{4}$, S. Yavuz ${ }^{2}$ ${ }^{1}$ Internal Medicine; ${ }^{2}$ Rheumatology; ${ }^{3}$ Cardiology; ${ }^{4}$ Biochemistry, Istanbul Bilim University, Istanbul, Turkey

Background: TWEAK is a type 2 transmembrane glycoprotein of TNF family that has multiple functions such as angiogenesis, regulation of tissue production- 
destruction, proinflammatory cytokine release. TWEAK and Fn14 receptor interaction has an important role in pathogenesis of atherosclerosis. Our aim was to evaluate subclinical atherosclerosis and its association with serum STWEAK, Fn14 and CD163 levels in RA patients.

Methods: One hundred Rheumatoid Arthritis (RA), 50 Spondyloarthritis (SpA) patients and 50 healthy controls $(\mathrm{HC})$ were included in the study. Serum soluble TWEAK, CD163 and Fn14 levels were measured by ELISA tecnique. Subclinical atherosclerosis was evaluated by echocardiography, which includes carotis intima media thickness (cIMT), aortic strain, stiffness and elasticity.

Results: In RA group 73 (73\%) patients were using DMARD's and 27 (27\%) were biological drugs, the mean DAS28 score was $3.49 \pm 1.31$. At the end of the study, TWEAK levels were found significantly lower (RA; $942.4 \pm 305.9$, AS; $1087.2 \pm 311.0$ and $\mathrm{HC} ; 1061.3 \pm 402.8 \mathrm{pg} / \mathrm{ml}, \mathrm{p}=0.05)$, and $\mathrm{Fn} 14$ levels were significantly higher (RA; $509.4 \pm 861.4$, AS; $163.5 \pm 249.0$ and $\mathrm{HC} ; 91.5 \pm 67.9 \mathrm{pg} / \mathrm{ml}, \mathrm{p}<0.01$ ) in RA patients compare to both groups. In addition Fn14 levels were higher in RA patients using biological drugs and seropositive subgroups (1042 \pm 1363 vs. $312 \pm 451 \mathrm{pg} / \mathrm{ml}, p<0.01$ and $674 \pm 101$ vs. $188 \pm 236 \mathrm{pg} / \mathrm{ml}$, respectively, $p<0.01$ ). Fn14 levels were correlated with disease duration $(r=0.38, p<0.01) . C D 163$ levels were similar in all groups. Although there was not any difference in cIMT measurements among groups, aortic stiffness was increased ( $p=0.03$ ), aortic strain and elasticity were decreased $(\mathrm{p}=0.03, \mathrm{p}=0.02)$ in RA patients compare to healthy controls. Multivariate analysis showed cardiovascular parameters were only associated with age.

Conclusions: In this study we observed impaired aortic parameters showing subclinic atherosclerosis in RA patients. At the same time, decreased levels of serum STWEAK and elevated levels of serum SFn14, were also shown in RA group. The difference in levels of serum soluble form of these biomarkers may be related to vascular damage in our RA patients who have low disease activity. However, further studies are needed to demonstrate association between atherosclerosis and these biomarkers in RA patients.

Disclosure of Interest: None declared

DOI: 10.1136/annrheumdis-2017-eular.2267

\section{AB1047 CAN THE ACR/EULAR 2010 CLASSIFICATION CRITERIA BE USED AS DIAGNOSTIC TOOL FOR RHEUMATOID ARTHRITIS IN REAL LIFE?}

N.F.L. Conijn ${ }^{1}$, D. Lopes Barreto ${ }^{1}$, T.M. Kuijper ${ }^{1}$, M. van der Steen $^{2}$, J. van der Kooij ${ }^{3}$, A.E.A.M. Weel ${ }^{1}$, J.M.W. Hazes ${ }^{4}$, M.R. KoK ${ }^{1} .{ }^{1}$ Rheumatology; ${ }^{2}$ Business intelligence; ${ }^{3}$ Planning and Control, Maasstad hospital; ${ }^{4}$ Rheumatology, Erasmus Medical Center, Rotterdam, Netherlands

Background: To date, due to the large variability in its clinical manifestations, early identification of rheumatoid arthritis (RA) relies on practice-based evidence. Moreover, this variation hampers the comparability and accurate stratification of the base population within and between RA trials. In 2010 the ACR/EULAR working group developed RA classification criteria that were primarily intended for research purposes. Despite its use in scientific settings, one can speculate on the effectiveness of these criteria when used in the routine clinical practice of diagnosing RA.

Objectives: In this study we aimed to investigate the degree of concordance between the diagnosis of RA in routine clinical practice and the ACR/EULAR 2010 classification criteria.

Methods: All patients who received a diagnosis of RA between 2010-2016 within our hospital were identified according to the financial diagnosis treatment combination (DTC) code, which corresponds to the ICD-10. Clinical and demographic data were extracted from our digital patient records of which $10 \%$ of the data were cross-checked by random selection. In retrospect we collected variables at time of RA diagnosis such as: number and type of swollen/painful joints, inflammatory markers, rheumatoid factor (RF), anti-citrullinated protein antibody (ACPA), disease duration and patients primary/secondary/tertiary diagnosis according to the rheumatologist. Additionally, all patients were classified according to the ACR/EULAR 2010 criteria for RA. The degree of concordance was determined by descriptive statistics.

Results: The database included 977 patients with a DTC RA of which 673 (69\%) had RA according to the rheumatologist. From the patients who were clinically diagnosed with RA, $463(69 \%)$ fulfilled the ACR/EULAR 2010 criteria (see figure 1), this is $47 \%$ of the total DTC RA patients. The majority of the population was female $(72 \%)$ and the mean age was 59 . A number of $161(24 \%)$ patients were diagnosed with RA according to the rheumatologists, but did not fulfil the ACR

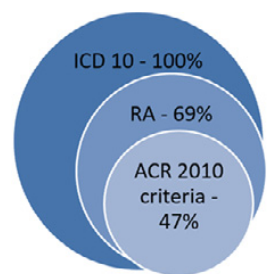

Fig. 1. Degree of concordance between RA identification and diagnosis, and classification criteria RA refers to the diagnosis RA made by the rheumatologist. As some patients with RA may have been incorrectly classified by ICD 10 , the circles are not completely overlapping. criteria. These patients had less inflammation, were more often RF and/or ACPA negative, and had less involved joints. About $5 \%$ of the data were missing. Conclusions: It can be concluded that the DTC codes are not the most reliable source of information in terms of the diagnosis. There is a discrepancy between the DTC code, the diagnosis according to the rheumatologist and the classification criteria. The degree of concordance between rheumatologist and the ACR criteria is comparable to the numbers described in literature. Since in our practice aspects of the ACR classification are used for diagnostic purposes, we will investigate factors that drive the specificity. Furthermore, reasons for the ICD-10/ DTC and final diagnostic mismatch is of great importance and will be studied as well. These factors will indicate opportunities on the use of the ACR/EULAR criteria in clinical practice. Acknowledgements: None

Disclosure of Interest: None declared

DOI: 10.1136/annrheumdis-2017-eular.5790

\section{AB1048 ULTRASOUND ASSESSMENT OF CARTILAGE THICKNESS IN PATIENTS WITH HAND OSTEOARTHRITIS}

O. Balueva ${ }^{1}$, A. Sarapulova ${ }^{2}$, O. Teplyakova ${ }^{2} .{ }^{1}$ Ultrasound Diagnosis, Medical Association "New hospital"; ${ }^{2}$ Urals State Medical University, Yekaterinburg, Russian Federation

Background: The destruction of cartilage is one of the basic mechanisms of the progression of hand osteoarthritis (HOA). Radiographic study indicates the thickness of the cartilage indirectly based on the degree of joint space narrowing (JSN). At the same time, the ultrasound (US) method allows to measure it directly. Objectives: To determine the thickness of the cartilage in the interphalangeal joints of patients with HOA and to correlate this rate with the degree of JSN, identified by radiologic imaging.c imaging.

Methods: 45 women at the age of $45-75$ years were included in the research. All of them were diagnosed of HOA according to the criteria of American College of Rheumatology (ACR). For each patient 8 proximal interphalangeal joints (PIP) were surveyed. The conventional radiographs (CRs) of each hand were performed for all patient in the standard frontal projection and the radiological changes were assessed using Kallman's method. Next, US investigation was carried out. The thickness of the cartilage was measured in the central part of the 2-5 proximal phalanx heads of both hands in the dorsal longitudinal view in the static image in gray- scale with the flexion position no more than 90 degrees. The cartilage is defined as a thin hypoechoic smooth layer, paralleling the contours of the articular surface. The thickness of the cartilage was defined as the distance between the subchondral bone and the surface layer of cartilage, which was the border between the cartilage and the joint cavity. In the presence of synovitis measurement of joint cartilage was carried out before the border and hypertrophic synovial if they differed in the degree of intensity of the US signal. The measurement of cartilage thickness was carried out by a conventional line, which was an exact perpendicular to the surface of the subchondral bone and parallel to the direction of US waves. Measurement was not carried out if visualization of the surface layer of cartilage was not possible. The control group consisted of 45 women $45-75$ years old without HOA, but with ultrasound investigation. Data were analyzed using Statistica 10.0 and are presented as mean (standard deviation), "odds ratio" coefficient (OR) and $x 2$ Pearson's correlation coefficient. Results: 360 joints of both hands were investigated with successful measurement of the cartilage thickness of $338(94.7 \%)$ joints. The average value of the cartilage in all PIP was $0.31(0.11) \mathrm{mm}$. The maximum thickness of the cartilage was obtained in the 2nd PIP joints - $0.34(0.12) \mathrm{mm}$ on the right hand and $0.36(0.14)$ $\mathrm{mm}$ on the left hand. For the control group results were $0.38(0.11) \mathrm{mm}$ and 0.37 $(0.13) \mathrm{mm}$ respectively. We have found a reliable relationship between the degree of JSN and cartilage thickness with the OR 1.849 (95\% confidence interval 1.198 $-2.855), x^{2}=7.772, p=0.0053$

Conclusions: US reduction of the cartilage thickness is a marker of cartilage loss which is correlated with the results of radiologic examination. This makes possible to use ultrasound as an alternative method of diagnosing HOA.

Disclosure of Interest: None declared

DOI: 10.1136/annrheumdis-2017-eular.6512

\section{AB1049 CLINICAL UTILITY OF AUTOANTIBODIES AGAINST EXTRACTABLE NUCLEAR ANTIGENS IN ROUTINE CARE: FREQUENCY OF REPEATED TEST REQUESTS AND DIAGNOSTIC VALUE OF ANTI-JO-1 (ANTI-HISTIDYL-TRNA SYNTHETASE)}

P. Jobanputra ${ }^{1}$, F. Malik ${ }^{2}$, E. Derrett-Smith ${ }^{1}$, T. Plant ${ }^{3}$, A. Richter ${ }^{4} .{ }^{1}$ Department of Rheumatology, Queen Elizabeth Hospital Birmingham, Birmingham; ${ }^{2}$ Department of Rheumatology, Epsom \& St. Helier University Hospitals NHS Trust, Carshalton; ${ }^{3}$ Department of Immunology, University of Birmingham; ${ }^{4}$ Department of Immunology, Queen Elizabeth Hospital Birmingham, Birmingham, United Kingdom

Background: High-throughput high-sensitivity ELISAs for autoantibodies associated with CTD, such as extractable nuclear antigens (ENA), are used widely. Anti-Jo-1 (anti-histidyl-tRNA synthetase), one of this panel, is believed to confer a poor prognosis due to an association with interstitial lung disease (ILD) and myositis. 